\title{
Lynch Syndrome
}

National Cancer Institute

\section{Source}

National Cancer Institute. Lynch Syndrome. NCI Thesaurus. Code C8494.

An autosomal dominant hereditary neoplastic syndrome characterized by the development of colorectal carcinoma and a high risk of developing endometrial carcinoma, gastric carcinoma, ovarian carcinoma, renal pelvis carcinoma, and small intestinal carcinoma. Patients often develop colorectal carcinomas at an early age (mean, 45 years). In the majority of the cases the lesions arise from the proximal colon. At the molecular level, high-frequency microsatellite instability is present. 